\section{TUMOUR SUPPRESSION}

\section{No contact please}

The naked mole rat exhibits resistance to cancer and is extremely longlived. The cancer resistance results from an enhanced form of contact inhibition, early contact inhibition (ECI), which is induced when naked mole rat cells are at a lower density than mouse cells. However, what triggers ECI in the naked mole rat has remained unknown.

Conditioned media from naked mole rat fibroblast cultures is more viscous than that from cultures of cells from mouse, human or

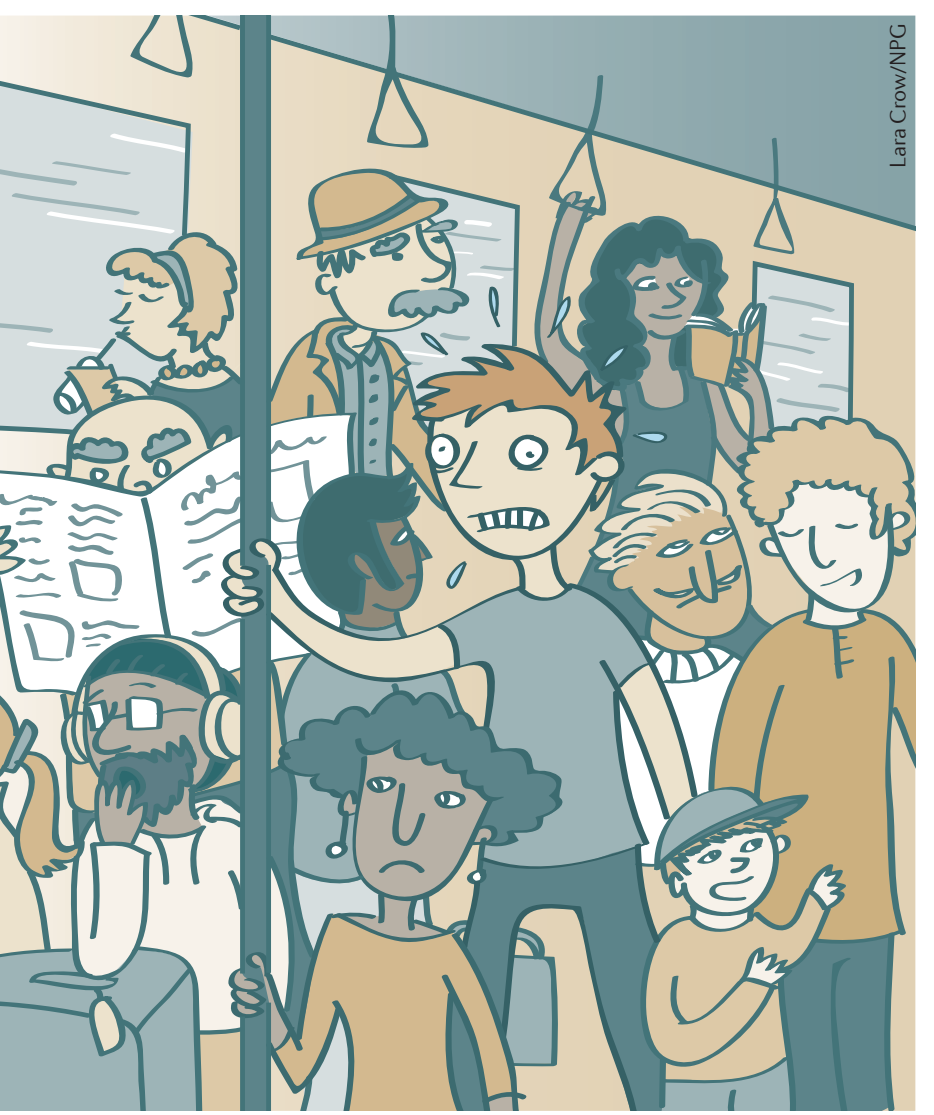

guinea pig (the closest phylogenetic relative to the naked mole rat), so the authors, led by Seluanov and Gorbunova, surmised that a secreted substance might induce ECI. They found that high-molecular-mass hyaluronan (HMM-HA) accounted for the high viscosity of naked mole rat fibroblast-conditioned media. HA is a component of the extracellular matrix (ECM), and HA polymer length modulates the cellular response on HA binding.Importantly, degradation of HMM-HA allowed naked mole rat fibroblasts to grow at a higher density than when HMM-HA was present in the culture medium, indicating that HMM-HA induces ECI.

HA synthase 2 (HAS2) produces HMM-HA, and the authors showed that this enzyme is overexpressed in naked mole rat fibroblasts compared with mouse and human fibroblasts and compared with naked mole rat embryonic fibroblasts (that do not undergo ECI). Furthermore, the authors confirmed that many naked mole rat tissues express HMM-HA, unlike mouse or guinea pig tissue sections. The authors also found that naked mole rat cells express a much lower activity of hyaluronidase (which degrades HA), and naked mole rat fibroblasts had a higher affinity for HA binding than did human or mouse cells. Together, this indicates that increased production and reduced degradation of HMM-HA results in high levels of HMM-HA in the ECM and that naked mole rat fibroblasts have a high sensitivity to HMM-HA.
So, how does HMM-HA induce $\mathrm{ECI}$ ? CD44 is a receptor for HA, and the authors showed that when CD44 was blocked using antibodies naked mole rat fibroblast cultures reached a high density. Intracellullarly, CD44 interacts with merlin, and ECI induction results in unphosphorylated (growthinhibitory) merlin and induces the expression of the cyclin-dependent kinase inhibitor INK4A (which is known to induce ECI).

What about cancer resistance? Naked mole rat fibroblasts do not undergo transformation induced by HRAS-G12V and SV40 large $\mathrm{T}$ antigen, which is indicated by a failure to grow in soft agar. However, when HMM-HA was degraded or CD44 was blocked, naked mole rat fibroblasts formed colonies in soft agar. Finally, the authors generated stably transformed naked mole rat fibroblasts by suppressing HAS2 expression or by overexpressing an HA-degrading enzyme, and these cells formed tumours in mice, unlike untransformed naked mole rat fibroblasts. Therefore, HMM-HA is an important suppressor of naked mole rat fibroblast transformation; it remains unclear whether this affects epithelial cell transformation.

The authors speculate that the production of HMM-HA by naked mole rats may have evolved as an adaptation to living in tunnels such that HMM-HA may confer a more flexible skin. However, this adaptation also seems to have conferred the cancer resistance exhibited by naked mole rats, which may also contribute to their longevity.

Gemma K. Alderton

ORIGINAL RESEARCH PAPER Tian, X. et al. High-molecular-mass hyaluronan mediates the cancer resistance of the naked mole rat. Nature http://dx.doi.org/10.1038/nature12234 (2013) 